\title{
Towards Designing an Assistant for Semi-Automatic EMS Dispatching
}

\author{
Melanie Reuter-Oppermann \\ Karlsruhe Service Research \\ Institute, Institute of Operations \\ Research, \\ Karlsruhe Institute of Technology \\ melanie.reuter@kit.edu
}

\author{
Stefan Morana \\ Karlsruhe Service Research \\ Institute, Institute of Information \\ Systems and Marketing, \\ Karlsruhe Institute of Technology \\ stefan.morana@kit.edu
}

\author{
Peter Hottum \\ Karlsruhe Service Research \\ Institute, \\ Karlsruhe Institute of Technology \\ peter.hottum@kit.edu
}

\begin{abstract}
Many Emergency Medical Service (EMS) systems worldwide handle emergency rescues as well as patient transports and dispatchers need to assign ambulances to incidents manually throughout the day. The management of the complex system together with the manual assignments can easily create stress for and pressure on the dispatchers. Mathematical algorithms can help improving the dispatching quality, but then dispatchers still need to choose the best-fitting algorithm and furthermore, trust the algorithm's dispatching suggestion. We propose an assistant that can support the EMS dispatchers. The assistant offers explanations for the choice of the algorithm as well as the dispatching suggestion in order to increase the dispatchers' trust and decrease their stress. We ground the assistant's design in Information Systems as well as Operations Research literature and thus, show how interdisciplinary service research can contribute in designing artefacts for complex service systems to solve real-world problems.
\end{abstract}

\section{Introduction}

Emergency Medical Service (EMS) systems throughout the world share the same goal: helping patients as fast as possible in case of an emergency. While emergency rescue is the main task, timeuncritical patient transports to, from and between hospitals are also an important service for many EMS systems. Regularly, the control center faces the general issue of having only a limited number of ambulances for a dedicated region to fulfill the services.

Within these complex systems, centralized dispatchers need to make the decision which ambulance should be assigned to which incident. In many countries worldwide, this management task is still done manually by the dispatcher possibly resulting in non-optimal assignments. In order to increase the probability for patient survival and recovery, especially emergency rescues demand for optimal assignments.

For many countries, thresholds on the maximum response time exist. Often, EMS laws state that for a certain percentage of emergency calls an ambulance must arrive within a predefined time. Due to cost pressures in the healthcare sector, the number of ambulances that can be used within a region is strictly limited. Accordingly, ambulances need to be located in such a way that the response time requirements can be fulfilled. While in case of life-threatening emergencies usually the closest ambulance is assigned to the call, the dispatching of ambulances to non-critical calls might also take the (current) number and location of further available ambulances into account.

Operations Research (OR) can not only help finding optimal locations for the ambulances (and relocate them over the day if applicable) [8], but also dispatch ambulances to emergencies [7], or schedule patient transports in order to minimize the tardiness of the transports [38].

For all these problems, various models and algorithms have been presented that incorporate different constraints and objectives. Furthermore, they differ in the time needed to determine either optimal or approximate solutions (in case heuristics are applied). Therefore, the pure application of such algorithms, e.g. by implementing them in a dispatching software, is not fully solving the overall issue. First, there is no one-fitsall-algorithm that addresses all possible problems and contexts in ambulance management. In case of an emergency rescue, minimizing the response time is the ultimate goal. In contrast, for a time uncritical patient transport minimizing the travel distances or late arrivals might be the aim. Thus, we argue that the dispatcher needs to select the appropriate algorithm for the context of the dispatching task.

Proposing that leads to the next issue as dispatchers are usually not able to understand and select the appropriate algorithm for the given task due to a lack of knowledge in OR. Algorithms in OR are complex and the selection of the best-fitting algorithm - including the 
suitable constraints and objective(s) - for a given context requires an in-depth understanding of the algorithms. Moreover, in case of emergency rescues, the dispatcher needs to make time-critical decisions and may be subject to continuous stress. Similarly, the required cognitive effort to make the dispatching is high given the EMS context.

Supporting individuals in decision-making and the selection of a certain item is investigated in Information Systems (IS) research in the context of decision support systems (DSS) and recommender systems (RS). These systems support individuals, for example, in deciding which display format fits best for a given situation [40] or deciding which camera fits best in a shopping agent [46]. The decision and recommendation can also be grounded by such a system via the provision of explanations [17] increasing the individuals acceptance [50], trust, and adoption [46] of the recommendation or decision, while at the same time reduce the users' cognitive effort [48].

We argue that a dispatching software should use multiple algorithms that are then selected and combined in their best way based on the current context. In order to support the dispatcher in the proper selection of the algorithms we propose an assistant system grounded in DSS and RS research for two reasons. Firstly, we combine those research fields (IS and OR) to solve our general problem in the domain of EMS. Secondly, we generalize the proposed solution to the more abstract problem class [18], enabling users with limited expertise to utilize expert knowledge or advanced functionality, e.g. OR algorithms.

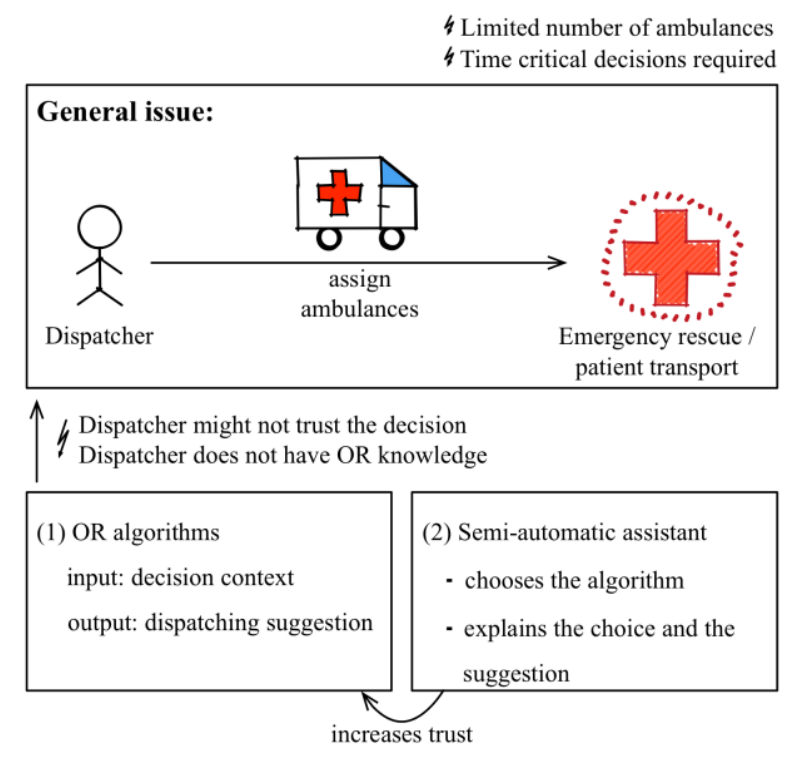

Figure 1. Summary of the content

Figure 1 summarizes the content of this work: dispatchers need to assign ambulances to emergency rescues and patient transports resulting in a very high workload and possibly stress. OR algorithms can be applied to determine assignment suggestions, but dispatchers might not trust the algorithms, also due to a probably lack of OR knowledge. A semi-automatic assistant can help increase the trust by explaining the suggestions. Our research addresses the following research question:

How to design an assistant for supporting the selection of dispatching algorithms in order to increase the individuals' acceptance of as well as trust in the assistant, decreasing the users' cognitive effort, and increasing the decision quality?

To answer the question we combine knowledge from the areas of OR as well as IS to investigate the phenomena in an interdisciplinary approach as outlined in the following. Hereby, we focus on the design of an assistant to support the dispatcher managing complex service system in an interdisciplinary approach [32]. Based on Ostrom et al. [33] we involve "multidisciplinary teams with different approaches, [as] Service design is one of the areas in which the support of interdisciplinary dialogues and integration of theories is crucial". By doing so we show that interdisciplinary service research can successfully be applied to solve real-world problems.

\section{Foundations and Related Work}

\subsection{Operations Research Literature}

Emergency Medical Service (EMS) systems as they exist worldwide are very complex. While there are differences between the systems, they share one goal: to serve as many emergencies as possible within the maximum response time (given by law). Besides the time-critical emergency rescues, many EMS systems also handle transports of patients to, from, or between hospitals. These transports are usually not time-critical and can often be scheduled. Either one set of ambulances is used for both services or two distinct sets exist. Then the dispatching of emergency ambulances is in independent of the dispatching of transportation ambulances, as patient transportation ambulances are usually not suitable for emergency rescues and using an emergency ambulance for patient transports lead to unnecessary cost and a decrease of coverage.

Still the most common dispatching rule in many EMS systems is to assign the closest idle ambulance to (life-threatening) emergencies, even though Carter et al. [10] already showed that this approach is not always optimal for the system. Obviously, it is important for life-threatening emergencies to arrive at the scene as fast as possible. For some countries, an EMS law even 
demands this. Dean [11] studied this dispatching rule and found that implementing also other rules could improve the overall response time performance.

Figure 2 gives an example for the dispatch policies. If in part 1 the emergency E1 that emerges first is lifethreatening, ambulance A2 will be assigned as it is the closest idle one. This means that emergency E2 that emerges after E1 cannot be reached by an ambulance within the maximum response time as ambulances $\mathrm{A} 1$ and $\mathrm{A} 3$ are further away. If the emergency is not lifethreatening, it is also possible to assign ambulance A1 that can arrive at E1 within the defined maximum response time. Then, emergency E2 can be served by ambulance A2 and in both cases an ambulance arrives within the maximum response time (part 2 of Figure 2).

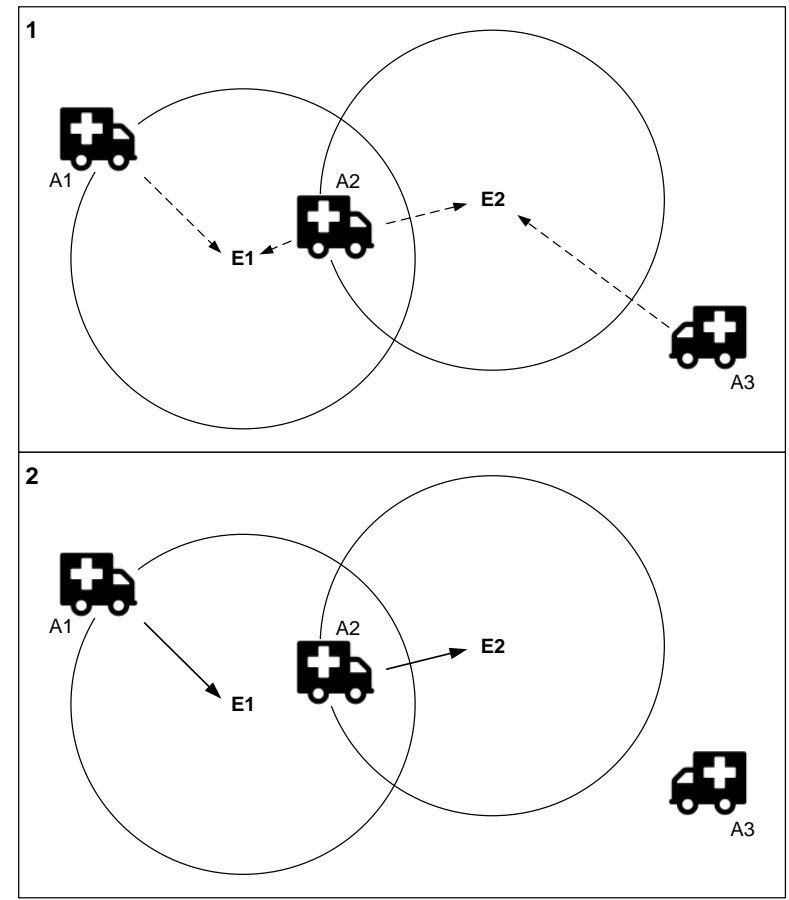

Figure 2. Example dispatch policy

Only few researchers have studied other dispatching rules. Andersson and Värbrand [3] adopted alternative dispatch rules for low priority calls while they do not look for optimal dispatching rules. Schmid [39] used approximate dynamic programming to find dispatch policies and showed that deviating from the closest idle rule for non-life-threatening calls can improve the overall performance. Recently, Jagtenberg et al. [24] have presented a dispatching heuristic that significantly reduced the fraction of late arrivals in their study with the drawback of an increased average response time.

Patient transports can be either scheduled in advance or assigned to ambulances immediately before the transport needs to take place. This depends on the policy of the EMS system as well as on the point of time the transportation task emerges. Parragh et al. [34] introduce formulations and solution approaches for the static patient transportation problem with different types of ambulances. Of course, solving a mathematical formulation to optimality can only be applied in practice if all patient transport tasks are known in advance, e.g., the night before, and if the problem size allows for a solution in acceptable time. If this is not the case, heuristics are necessary in practice. If none or only part of the tasks are known in advance and the rest becomes known throughout the day, scheduling the tasks resembles an online problem. Ardekani et al. [4] present three heuristics for the patient transportation problem: a simple heuristic that either can be used directly or to determine a starting solution for the more advanced second heuristic that further improves the solution. The third heuristic inserts short-term demand into the existing schedule in real-time. For the online case, but when rescheduling is possible, Kergosien et al. [26] propose a tabu search heuristic, which is called every time a new transport emerges. Schilde et al. [38] study the problem of incorporating unknown but expected return transports for the patients. They model it as a dynamic stochastic problem and propose four advanced variants of metaheuristics. While the presented results are promising, the problem will not be applicable for all EMS systems and the approaches might also be too complex for some users.

Many papers study either emergency rescues or patient transports, only few investigate both problems simultaneously. Kergosien et al. [25] built a generic discrete event simulation-based analysis model that studies the management of a fleet of ambulances with the aim of optimally serving emergency requests as well as transporting patients between their homes and hospitals. Note that for this paper we make two assumptions:

(1) Two distinct sets of ambulances are used for emergency rescues and patient transports leading to disjoint sets of algorithms for both services.

(2) In case of life-threatening emergencies, always the closest idle ambulance is assigned.

Due to the first assumption, patient transports and (non-life-threatening) emergencies demand for different dispatching algorithms. Depending on the objectives and the current point in time, varying algorithms can be necessary and useful for both problems. This means that for each incoming call one out of multiple algorithms has to be chosen in order to efficiently dispatch an ambulance to the incident. 


\subsection{Information Systems Literature}

In IS research, there exist various concepts and classes of systems aiming to support users with making decisions. One class of such IS are DSS that aim to provide decisional advice [45] to enable faster, better, and easier decision making. DSS are applied, for example, for a medical diagnosis [9], or supervising a nuclear power plant [31]. In order to explain to the user why the system performs a certain action, suggests a specific decision, or outputs a final result, DSS provide 'decisional guidance' [41]. Another class of systems are Expert Systems (XPS) that aim to support humans with their decision making process by emulating the decision-making ability of a human expert [23]. XPS and the related Knowledge-Based Systems (KBS) guide humans through complex decision problems, using an integrated knowledge base. A more modern class of systems support users' decision-making are RS based on the previously collected and aggregated recommendations from other humans [36]. The main feature of RS is the knowledge base that is developed by either explicitly asking the users for their preferences [35], or learning from their prior usage behavior. We summarize all these systems that aim to support users' decision-making under the term 'decision aids' for brevity, acknowledging that these are actually all distinct research streams. Decision aids integrate the expertise of one or more experts in a given decision domain and intended to provide a specific recommendation to a given problem and/or provide expert advice that assists the user in making a better decision than when unaided [6].

In order to increase the users' acceptance of the provided decisions, suggestions, or results [50], decision aids can provide explanations [37, 17] that describe what the system knows, how it works, and why specific actions are appropriate [42]. Decision aids are implemented for many contexts and the positive effect on the users' acceptance and adoption is shown, for example, in the context of online shopping and ecommerce $[2,46]$. Related to the users' trust into the provided support is the trust into provided support. Researchers investigate the effect of explanations on trust building in the context of decision aids [46, 47]. Decision aids are also implemented for other context. $\mathrm{Li}$ and Gregor [29] investigate online advisory services, a form of decision aids, with a build in explanatory facility. Their findings show that the explanations provided by the explanatory facilities result in an improved decision-process satisfaction and decisionadvice transparency [29]. Decision aids are also investigated in the context of emergency management information systems. Shen et al. [40] conduct two experiments and show that decision makers tend to not choose the most appropriate display format. In contrast to this, when users are supported in their decision making by provided decisional guidance, their performance, measured as decision accuracy as well as decision speed, increases [40].

In summary, the research on decision aids in IS research is a sound grounding for addressing our research question. The existing research on how to support users' decision making in order to improve the decision accuracy while at the same time ensuring the users' acceptance and trust of the decision is applied for the grounding of the dispatch assistant design.

\section{Design Science Research Project}

Design Science Research (DSR) aims to design a solution for a given class of problems [18, 22]. Moreover, DSR aims to balance rigor by following established research methods as well as incorporating existing theoretical knowledge and relevance by addressing practically motivated issues as well as evaluating the design outcome in the practice [21]. We argue that the support of EMS dispatchers in the selection of the proper algorithm is an important practical issue. The OR literature shows that, depending on the context and the objective, there are several suitable algorithms. However, the selection of the bestfitting algorithm requires both, an understanding of the context (given for the dispatchers) and the algorithm itself (lacked by the dispatchers). Moreover, usually a dispatcher needs to decide on the ambulance assignment immediately in case of an emergency. This situation can result in an increased stress level for the dispatcher and increases the possibility for making wrong decisions. From a more abstract point of view, the selection of the proper algorithm is a decision task and there is valuable research available to support individuals' decisionmaking. In order to address this problem, we started a DSR project to design an assistant system supporting EMS dispatchers in the selection of the best-fitting algorithm. We decided to apply the DSR approach due to the high practical relevance of the addressed problem and the existing theoretical knowledge on supporting individuals' decision-making.

For the DSR project, we choose a setting with three collaboration partners: an EMS software company, a software company that implements the algorithms, and dispatch centers of several EMS regions. The first software company gives access to the input data and the interface in order to execution the dispatching suggestion if the assistant accepts it. The second company implements the algorithms and connects the tool to the EMS software via the given interface. The assistant as the user's interface chooses the best-fitting 
algorithm as well explains the choice and the resulting dispatching advice. Figure 3 displays the general structure of the three components and the flow of information between them.

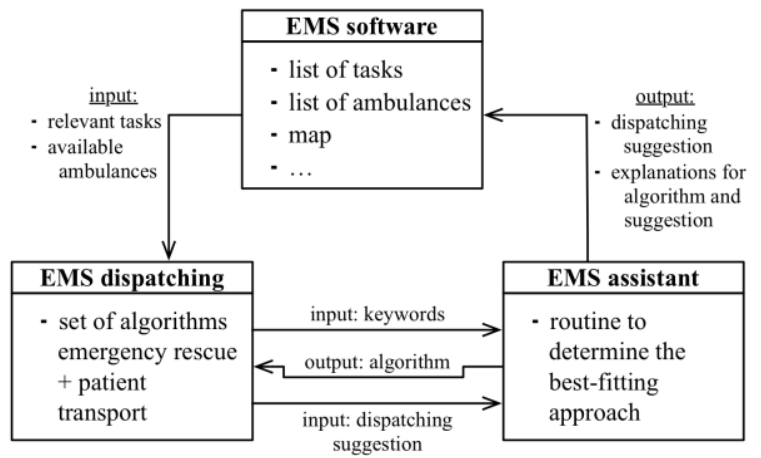

Figure 3. Structure of the components

Our DSR project follows the suggestions by Kuechler and Vaishnavi [28] and is currently between the stages problem awareness and the suggestions of the design. In the following section, we outline the design of the EMS assistant grounded in OR and IS literature. Subsequently, we briefly sketch the planned evaluation of our design.

\section{Designing a Semi-Automatic Assistant}

We are currently in the stage of deriving the theorygrounded design principles for EMS assistants. In the following section, we briefly discuss the current version of the two design principles (DP1 and DP2) with respect to the underlying OR and IS literature.

DP1: Semi-automatic ambulance dispatching based on mathematical algorithms

The selection of a dispatching algorithm is based on the users' input and the actual context. The first important keywords are emergency rescue and patient transport. Based on these keywords, the request category is determined. For both categories, disjoint sets of algorithms are available. Additional input parameters for choosing the best-fitting algorithm are the emergency level for emergency rescues and the pick-up time for patient transports.

We argue the implemented EMS is context-aware [1]. It monitors the calls and is able to detect keywords based on speech-to-text recognition. The information is then used by the EMS assistant to pre-select certain input parameters for the recommendation.

Table 1 shows the possible dispatching algorithms for emergency rescues. As mentioned above, for lifethreatening emergencies always the closest idle ambulance is assigned to minimize the response time for these incidents individually. For all other emergency incidents, different heuristics can be applied, depending also on the chosen objective.

Table 1. Emergency rescue

\begin{tabular}{|l|l|l|}
\hline Incident & Objective & Method \\
\hline $\begin{array}{l}\text { Life- } \\
\text { threatening }\end{array}$ & $\begin{array}{l}\text { Min response } \\
\text { time }\end{array}$ & $\begin{array}{l}\text { Closest idle } \\
\text { policy }\end{array}$ \\
\hline \multirow{4}{*}{$\begin{array}{l}\text { Non-life- } \\
\text { threatening }\end{array}$} & $\begin{array}{l}\text { Min average } \\
\text { response time }\end{array}$ & $\begin{array}{l}\text { Min overall } \\
\text { lateness }\end{array}$ \\
\cline { 2 - 2 } & $\begin{array}{l}\text { Dispatching } \\
\text { heuristic(s) } \\
\text { (e.g., [24]) }\end{array}$ \\
\cline { 2 - 3 } & $\begin{array}{l}\text { Min weighted } \\
\text { sum of both }\end{array}$ \\
\hline
\end{tabular}

To contrast this with regard to patient transportation requests, Table 2 outlines applicable methods and differentiations based on the time the request arrives in the call center. If requests are known the day before, they can already be planned before, using either an exact approach or a heuristic. For the heuristic, different options exist that vary in the complexity, solution quality and run time. Based on former experience with EMS call center managers, algorithms might need to be comparably easy to understand in order to trust their suggestions, while for others it is sufficient to know the general idea of the algorithm and the applied objective. It is also a strength of our approach that it actually allows the implementation of multiple alternative algorithms that can then be chosen by the particular dispatchers of each EMS region instead of developing individual tools for each region and thereby decreasing the development effort and increasing the usability for many EMS regions.

Even though tasks may be known in advance, it is also possible in practice that none of them is scheduled in advance, but only throughout the day. Then, an online approach can be applied. If more than only the next task is considered, this look-ahead can be incorporated into the decision-making and possibly improve the solution [13]. Demand that emerges only shortly before the pickup time can also be integrated by online approaches. If future tasks are already scheduled but can be rescheduled, if necessary, then heuristics can be used that deliver solutions in a matter of seconds.

For all approaches different objectives can be used (and therefore chosen by the dispatcher), either depending on the current situation or fixed by a general policy. For the provider minimizing the driving times is usually the main goal in order to minimize the cost for staff and vehicles. For the patients (as well as hospitals and practices), it is most important that the ambulance 
arrives in time. The corresponding objective minimizes the lateness of the ambulance arrivals at the pick-up applied. The intended assistant has to guide the dispatchers through the application of the available

Table 2. Patient transport

\begin{tabular}{|c|c|c|c|}
\hline Incident & Method & Characteristics & Objective \\
\hline \multirow{7}{*}{$\begin{array}{l}\text { Short-term } \\
\text { demand }\end{array}$} & \multirow{3}{*}{$\begin{array}{l}\text { Online dispatching } \\
\text { (e.g., [13]) }\end{array}$} & \multirow{3}{*}{$\begin{array}{l}\text { Simple and fast; best solution for a single } \\
\text { task; drawback on the system wide } \\
\text { performance }\end{array}$} & Min lateness \\
\hline & & & Min driving time \\
\hline & & & Min weighted sum of both \\
\hline & \multirow{4}{*}{$\begin{array}{l}\text { Heuristic (if } \\
\text { rescheduling is } \\
\text { possible) (e.g., [26]) }\end{array}$} & \multirow{4}{*}{$\begin{array}{l}\text { Varying complexity; fast; improvement of } \\
\text { the overall performance; changes in the } \\
\text { schedule necessary }\end{array}$} & Min lateness \\
\hline & & & Min driving time \\
\hline & & & Min changes \\
\hline & & & Min weighted sum \\
\hline \multirow{12}{*}{$\begin{array}{l}\text { Known the } \\
\text { day before }\end{array}$} & \multirow{3}{*}{$\begin{array}{l}\text { Online dispatching } \\
\text { (e.g., }[13])\end{array}$} & \multirow{3}{*}{$\begin{array}{l}\text { Simple and fast; best solution for a single } \\
\text { task; drawback on the system wide } \\
\text { performance }\end{array}$} & Min lateness \\
\hline & & & Min driving time \\
\hline & & & Min weighted sum of both \\
\hline & \multirow{3}{*}{$\begin{array}{l}\text { Simple heuristic } \\
\text { (e.g., [4]) }\end{array}$} & \multirow{3}{*}{$\begin{array}{l}\text { Simple and fast; easy to explain; drawback } \\
\text { on the solution quality }\end{array}$} & Min lateness \\
\hline & & & Min driving time \\
\hline & & & Min weighted sum of both \\
\hline & \multirow{3}{*}{$\begin{array}{l}\text { Advanced heuristic } \\
\text { (e.g., [4]) }\end{array}$} & \multirow{3}{*}{$\begin{array}{l}\text { More complex; difficult to explain; still } \\
\text { fast; improved solution quality }\end{array}$} & Min lateness \\
\hline & & & Min driving time \\
\hline & & & Min weighted sum of both \\
\hline & \multirow{3}{*}{$\begin{array}{l}\text { Exact solution (e.g., } \\
[34])\end{array}$} & \multirow{3}{*}{$\begin{array}{l}\text { Optimal solution; time consuming; use of a } \\
\text { (commercial) solver or complex solution } \\
\text { method necessary }\end{array}$} & Min lateness \\
\hline & & & Min driving time \\
\hline & & & Min weighted sum of both \\
\hline
\end{tabular}

and/or drop-off locations. Often, a tradeoff between driving times and lateness is most relevant for practice. Then, a weighted sum of the two objectives can be used.

In IS research, there are also supportive findings on the positive effects of decision aids on the decision quality [43]. While the OR algorithms can determine good (or optimal) dispatching decisions, the user (i.e., the dispatcher) usually does not trust immediately a computed decision without further information. IS research has shown that decision aids with individual explanations can lead to a users' trust in and acceptance for the recommended decision $[46,50]$. In addition, explanations also decrease the user's cognitive effort [48], as he does not have to determine the best dispatching decision himself.

DP2: Provide explanations for semi-automatic ambulance dispatching

Providing explanations aims to increase the dispatchers' trust in the recommendation as well as their acceptance and adoption of the assistant itself [5, 50, 46]. To guarantee a recommended solution that takes into account all given regulations (such as time constraints) suitable dispatching algorithms have to be algorithms as well as the selection of the offered options. This independent guidance has to be provided by enabling the dispatcher in understanding the selected characteristics (see table 2) and their influence on the proposed result. Therefore, the assistant considers available information, collects necessary input, and generates appropriate dialogues to interact with the respective dispatcher.

Based on these input parameters the assistant is able to process the mathematical algorithms and the optimization internally with the goal to solve the given situation best with the given conditions. Furthermore, the presentation of the calculated solutions is enriched by human understandable explanations that enable the dispatcher to appreciate and accept the recommendation. Figure 4 depicts a mockup of the intended EMS assistant for an existing dispatching software.

In summary, we propose two theory-grounded design principles for an EMS assistant. The first design principle describes how the assistant supports the EMS dispatcher in the semi-automatic selection of the appropriate algorithm for the current decision context 
aiming to increase the decision quality regarding the defined objective. Design principle two states that the EMS assistant should provide explanations for the suggested algorithm and the dispatching decision in order in increase the users' intention to use the EMS assistant, increase the users' trust into the EMS assistant, and decrease the users' cognitive effort.

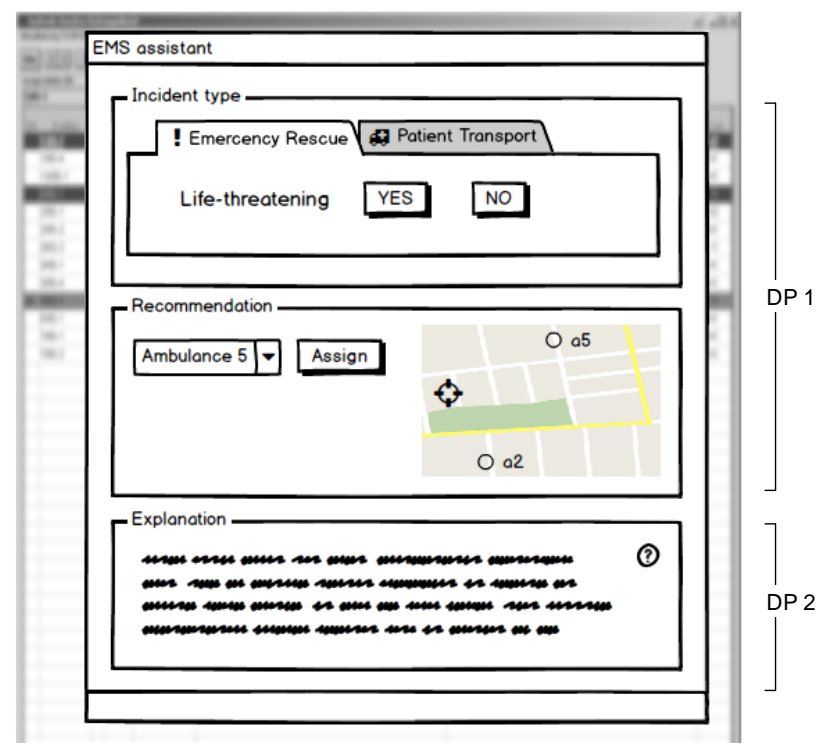

Figure 4. EMS assistant mockup

\section{Instantiation and Evaluation}

Following the DSR approach according to Kuechler and Vaishnavi [28], the next step after the design suggestions is the instantiation and evaluation of the design. In the following, we briefly outline the planned instantiation of the design as well as the evaluation.

For the instantiation of the EMS assistant design, we collaborate with several EMS dispatching centers and two software vendors developing the EMS software and the dispatching tool that contains the algorithms. The second software vendor will develop, in close cooperation with us, the EMS assistant and include it into the existing product that is again connected to the EMS software. As the EMS assistant is used in a critical environment, ultimately, the software is used to dispatch ambulances that are intended to save human lives. The software vendor will ensure the stability and correctness of both, the software and the underlying algorithms. This evaluation is out of scope for our research project, we will focus on the evaluation of the EMS assistant with respect to the validity of the suggested two design principles. The evaluation itself will be conducted in a controlled field setting with the actual software users (i.e., dispatchers).
As outlined in the previous section, we propose two design principles that influence, in total, four dependent variables. In order to test the proposed design, Gregor and Jones [19] suggest to formulate testable propositions. In the following, we discuss the testable propositions (P1 to $\mathrm{P} 4)$ and a research model for the evaluation of our design.

Research on decision aids in various contexts showed the positive effects of using a decision aids on the decision quality [20, 43, 49]. Following the existing findings, we argue that the usage of the EMS assistant, especially the semi-automated algorithm selection, will increase the decision quality with the respect to the current objective as formulated in:

P1: Using the EMS assistant increases the decision quality regarding the current objective.

Providing explanations in the context of decision aids has a long tradition in IS research and the existing empirical findings support the positive effects $[14,12$, $16,30,17,15,48]$. In the context of our research, we are especially interested in the effect of the explanations on the users' acceptance and trust of the provided recommendation as well as the effect of the users' cognitive effort. Researchers demonstrated the positive effect of providing explanations in order to increase the users' acceptance of the recommendation or more general the outcome of decision aids in various studies $[14,17,27]$. We argue that the provision of explanations by the EMS assistant on how the dispatching decision was made and why this is a good (or the best possible) solution, the users' acceptance of the dispatching is increased as formulated in:

P2: Using the EMS assistant and providing explanations increases the users' acceptance of the recommended dispatching solution.

Similar to the acceptance, the users' trust in the decision aids and their recommendation are subject to research in the IS domain [2, 46]. Especially in this critical context, the dispatching of ambulances, providing explanations on how the dispatching routing was done and why the presented solution is the best fitting solution will increase the users' trust into the EMS assistant as formulated in:

P3: Using the EMS assistant and providing explanations increases the users' trust into recommended dispatching solution.

The usage of decision aids can also affect the users' cognitive effort as the individuals cognitive capacity is eased $[48,44]$. Using the EMS assistant, the dispatcher can focus on the direct assignment of the ambulance as the assistant already calculated the best fitting dispatching routing for the current context. Accordingly, we argue: 
P4: Using the EMS assistant decreases the users' cognitive effort.

Figure 5 depicts our research model and the four testable propositions for the evaluation of our design.

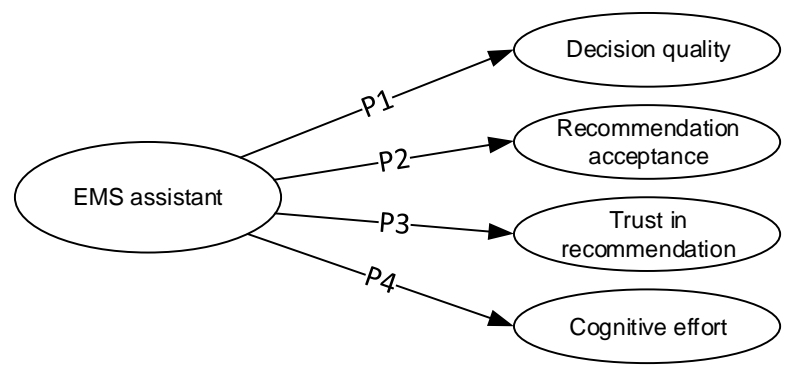

Figure 5. Research model

\section{Conclusion and Outlook}

This paper contributes to theory as well as practice by discussing an important challenge, the optimal dispatching of ambulances. Moreover, the related class of problems, i.e., the support of human decision making for limited resources in varying contexts, is investigated. We address this challenge in a DSR project that enables us to incorporate theoretical knowledge from OR as well as IS research and evaluate the resulting theorygrounded design in a real-world situation. In this paper, we provide the problem awareness as well as the initial suggestions for the theory-grounded design principles. In addition, we discuss the upcoming evaluation and present the evaluation model, grounded in existing theories from the IS literature. We propose the design of a semi-automatic assistant for ambulance dispatching in EMS systems. It chooses the best-fitting dispatching algorithm for the individual context, displays the suggestion and offers explanations for the choice of the algorithm as well as the suggestion for the dispatching. By combining knowledge and research principles from different fields, we show that interdisciplinary service research can successfully be applied to solve real-world problems in complex service systems [32, 33].

The dispatching decision becomes more complex when considering the need for more than one ambulance. In addition, in Germany the dispatcher also coordinates the emergency doctors, which usually arrive at the scene in separate vehicles. In addition, as ambulances can usually only transport one patient at a time, more than one ambulance must be dispatched to incidents involving multiple patients. Then, the dispatching problem gets more difficult and the decision complexity increases.

When the relocation of ambulances is part of the daily routine, the dispatchers also need to decide about those. Relocations can also be connected to the dispatching. While it is easier to only decide which base an ambulance is sent to after the patient was dropped off at the hospital, idle ambulances might actually be relocated to another location to improve the coverage of the considered region, for example when an ambulance was dispatched to an emergency and therefore left its base. Obviously, the workload for the dispatcher together with the decision complexity increases significantly. Then, the assistant could additionally propose relocations and explain possible options.

Future work could also include adding a forecast and prediction component into the EMS and the dispatching module. The forecast could include current and historic traffic situations, e.g. in order to avoid regular traffic jams in a city area. Moreover, the forecast could estimate future demand for regular ambulance transports as well as emergency rescues based on historic data. In doing so, the EMS dispatching itself could be further improved. Although, there are various opportunities for improving the dispatching using advanced analytical features, however, the human factor still needs to be considered. EMS dispatchers will require assistance in the utilization of these advanced analytical features, again, in order to ensure the individuals' trust as well as acceptances of the optimal dispatching.

\section{References}

[1] Abecker, A., A. Bernardi, K. Hinkelmann, O. Kuhn, and M. Sintek, "Context-Aware, Proactive Delivery of Task-Specific Information: The KnowMore Project", Information Systems Frontiers, 2(3/4), 2000, pp. 253276.

[2] Al-Natour, S., I. Benbasat, and R.T. Cenfetelli, "The Effects of Process and Outcome Similarity on Users' Evaluations of Decision Aids", Decision Sciences, 39(2), 2008, pp. 175-211.

[3] Andersson, T. and P. Värbrand, "Decision support tools for ambulance dispatch and relocation", Journal of the Operational Research Society, 58(S2), 2007, pp. 195-201.

[4] Ardekani, L.H., D. Haight, A. Ingolfsson, M. Salama, and M. Stanton, "Scheduling and routing ambulances that provide inter-facility patient transfers", working paper, 2014.

[5] Arnold, V., N. Clark, P.A. Collier, S.A. Leech, and S.G. Sutton, "The Differential Use and Effect of Knowledge-Based System Explanations in Novice and Expert Judgment Decisions", MIS Quarterly, 30(1), 2006, pp. 79-97.

[6] Arnold, V., P.A. Collier, S.A. Leech, and S.G. Sutton, "Impact of Intelligent Decision Aids on Expert 
and Novice Decision-makers' Judgments", Accounting and Finance, 44(1), 2004, pp. 1-26.

[7] Bandara, D., M.E. Mayorga, and L.A. McLay, "Priority dispatching strategies for EMS systems", Journal of the Operational Research Society, 65(4), 2014, pp. 572-587.

[8] Brotcorne, L., G. Laporte, and F. Semet, "Ambulance location and relocation models", European Journal of Operational Research, 147(3), 2003, pp. 451463.

[9] Buchanan, B.G. and E.H. Shortliffe, Rule-based expert systems: The MYCIN experiments of the Stanford heuristic programming project, AddisonWesley, Reading, Mass, 1984.

[10] Carter, G.M., J.M. Chaiken, and E. Ignall, "Response Areas for Two Emergency Units", Operations Research, 20(3), 1972, pp. 571-594.

[11] Dean, S.F., "Why the closest ambulance cannot be dispatched in an urban emergency medical services system", Prehospital and disaster medicine, 23(2), 2008, pp. 161-165.

[12] Dhaliwal, J.S. and I. Benbasat, "The Use and Effects of Knowledge-based System Explanations: Theoretical Foundations and a Framework for Empirical Evaluation", Information Systems Research, 7(3), 1996, pp. 342-362.

[13] Dunke, F., Online Optimization with Lookahead: Dissertation, Karlsruhe Institute of Technology (KIT), 2014.

[14] Giboney, J.S., S.A. Brown, P.B. Lowry, and J.F. Nunamaker, "User acceptance of knowledge-based system recommendations: Explanations, arguments, and fit", Decision Support Systems, 72, 2015, pp. 1-10.

[15] Gönül, M.S., D. Önkal, and M. Lawrence, "The Effects of Structural Characteristics of Explanations on Use of a DSS", Decision Support Systems, 42(3), 2006, pp. 1481-1493.

[16] Gregor, S., "Explanations From Knowledge-Based Systems and Cooperative Problem Solving: An Empirical Study", International Journal of HumanComputer Studies, 54(1), 2001, pp. 81-105.

[17] Gregor, S. and I. Benbasat, "Explanations from Intelligent Systems: Theoretical Foundations and Implications for Practice", MIS Quarterly, 23(4), 1999, pp. 497-530.

[18] Gregor, S. and A. Hevner, "Positioning and Presenting Design Science Research for Maximum Impact", MIS Quarterly, 37(2), 2013, pp. 337-355.
[19] Gregor, S. and D. Jones, "The Anatomy of a Design Theory", Journal of the Association for Information Systems:, 8(5), 2007, pp. 312-335.

[20] Häubl, G. and V. Trifts, "Consumer Decision Making in Online Shopping Environments: The Effects of Interactive Decision Aids", Marketing Science, 19(1), 2000, pp. 4-21.

[21] Hevner, A.R., "A Three Cycle View of Design Science Research", Scandinavian Journal of Information Systems, 19(2), 2007, pp. 87-92.

[22] Hevner, A.R., S.T. March, J. Park, and S. Ram, "Design Science in Information Systems Research", MIS Quarterly, 28(1), 2004, pp. 75-105.

[23] Jackson, P., Introduction to Expert Systems, Addison-Wesley, 1998.

[24] Jagtenberg, C.J., S. Bhulai, and van der Mei, R D, "Dynamic ambulance dispatching: is the closest-idle policy always optimal?", Health care management science, 2016.

[25] Kergosien, Y., V. Bélanger, P. Soriano, M. Gendreau, and A. Ruiz, "A generic and flexible simulation-based analysis tool for EMS management", International Journal of Production Research, 53(24), 2015, pp. 7299-7316.

[26] Kergosien, Y., C. Lenté, D. Piton, and J.-C. Billaut, "A tabu search heuristic for the dynamic transportation of patients between care units", European Journal of Operational Research, 214(2), 2011, pp. 442-452.

[27] Köhler, C.F., E. Breugelmans, and Dellaert, Benedict G. C., "Consumer Acceptance of Recommendations by Interactive Decision Aids: The Joint Role of Temporal Distance and Concrete Versus Abstract Communications", Journal of Management Information Systems, 27(4), 2011, pp. 231-260.

[28] Kuechler, B. and V. Vaishnavi, "Theory Development in Design Science Research: Anatomy of a Research Project", European Journal of Information Systems, 17(5), 2008, pp. 489-504.

[29] Li, M. and S. Gregor, "Outcomes of Effective Explanations: Empowering Citizens Through Online Advice", Decision Support Systems, 52(1), 2011, pp. 119-132.

[30] Mao, J.-Y. and I. Benbasat, "The Use of Explanations in Knowledge-Based Systems: Cognitive Perspectives and a Process-Tracing Analysis", Journal of Management Information Systems, 17(2), 2000, pp. 153-179.

[31] Mosier, K.L. and L.J. Skitka, "Human Decision Makers and Automated Decision Aids: Made for Each 
Other?", in Automation and human performance: Theory and applications, R. Parasuraman and M. Mouloua, Editors. 1996. Lawrence Erlbaum Associates: Mahwah, N.J.

[32] Ng, I.C., R. Maull, and N. Yip, "Outcome-based contracts as a driver for systems thinking and servicedominant logic in service science: Evidence from the defence industry", European Management Journal, 27(6), 2009, pp. 377-387.

[33] Ostrom, A.L., A. Parasuraman, D.E. Bowen, L. Patricio, and C.A. Voss, "Service Research Priorities in a Rapidly Changing Context", Journal of Service Research, 18(2), 2015, pp. 127-159.

[34] Parragh, S.N., J.-F. Cordeau, K.F. Doerner, and R.F. Hartl, "Models and algorithms for the heterogeneous dial-a-ride problem with driver-related constraints", OR Spectrum, 34(3), 2012, pp. 593-633.

[35] Pfeiffer, J., Interactive Decision Aids in ECommerce, Physica-Verlag HD, Heidelberg, 2012.

[36] Resnick, P. and H.R. Varian, "Recommender systems", Communications of the ACM, 40(3), 1997, pp. 56-58.

[37] Richardson, G.L., B.M. Jackson, and G.W. Dickson, "A Principles-Based Enterprise Architecture: Lessons From Texaco and Star Enterprise", MIS Quarterly, 14(4), 1990, pp. 385-403.

[38] Schilde, M., K.F. Doerner, and R.F. Hartl, "Metaheuristics for the dynamic stochastic dial-a-ride problem with expected return transports", Computers \& operations research, 38(12), 2011, pp. 1719-1730.

[39] Schmid, V., "Solving the dynamic ambulance relocation and dispatching problem using approximate dynamic programming", European Journal of Operational Research, 219(3), 2012, pp. 611-621.

[40] Shen, M., M. Carswell, R. Santhanam, and K. Bailey, "Emergency Management Information Systems: Could Decision Makers be Supported in Choosing Display Formats?", Decision Support Systems, 52(2), 2012, pp. 318-330.

[41] Silver, M., "Decisional Guidance for ComputerBased Decision Support", MIS Quarterly, 15(1), 1991, pp. 105-122.

[42] Swartout, W., "Explanation", in Encyclopedia of Artificial Intelligence, S.C. Shapirio and D. Eckroth, Editors. 1987: New York.

[43] Tan, W.-K., C.-H. Tan, and H.-H. Teo, "Consumerbased decision aid that explains which to buy: Decision confirmation or overconfidence bias?", Decision Support Systems, 53(1), 2012, pp. 127-141.
[44] Todd, P. and I. Benbasat, "Evaluating the Impact of DSS, Cognitive Effort, and Incentives on Strategy Selection", Information Systems Research, 10(4), 1999, pp. 356-374.

[45] Turban, E. and J.E. Aronson, Decision Support Systems and Intelligent Systems, 6th edn., Prentice Hall, Upper Saddle River, NJ, 2001.

[46] Wang, W. and I. Benbasat, "Trust In and Adoption of Online Recommendation Agents", Journal of the Association for Information Systems, 6(3), 2005, pp. 72-101.

[47] Wang, W. and I. Benbasat, "Recommendation Agents for Electronic Commerce: Effects of Explanation Facilities on Trusting Beliefs", Journal of Management Information Systems, 23(4), 2007, pp. 217-246.

[48] Wang, W. and I. Benbasat, "Interactive Decision Aids for Consumer Decision Making in E-Commerce: The Influence of Perceived Strategy Restrictiveness", MIS Quarterly, 33(2), 2009, pp. 293-320.

[49] Xiao, B. and I. Benbasat, "E-Commerce Product Recommendation Agents: Use, Characteristics, and Impact", MIS Quarterly, 31(1), 2007, pp. 137-209.

[50] Ye, L.R. and P.E. Johnson, "The Impact of Explanation Facilities on User Acceptance of Expert Systems Advice", MIS Quarterly, 19(2), 1995, pp. 157172. 